\title{
Reflexión sobre la gestión de innovación bibliotecológica: raíces, esencias, cambios
}

\author{
Emilio Setién Quesada \\ Biblioteca Nacional José Martí \\ La Habana, Cuba
}

\subsection{Resumen}

En breve recuento histórico se identifica la esencia del fenómeno bibliotecario en el complejo mundo de la información y sus disciplinas, y se reflexiona sobre algunos enfoques que ejemplifican las tendencias teóricas de la Bibliotecología contemporánea y sobre la globalización actual de la información, como premisas para enfrentar la innovación y la gestión de cambio que requiere la profesión hoy día. (Autor)

Palabras clave:Bibliotecología. Teoría. Paradigmas científicos. Historia. Globalización.

\subsection{Abstract}

Historical and theorethical overview of the essence of the library phenomenum in the context of the complex information world and its disciplines. (Author)

Keywords: Library science. Theory. Scientific paradigms. History. Gobalization.

\section{Introducción}

Los fundamentos de los cambios que reclama la innovación bibliotecológica en nuestros días se pueden abordar de múltiples formas, pero para enfrentar esa innovación, sin perder de vista el objetivo que le corresponde a la actividad bibliotecaria, es necesario volver a sus raíces para tener clara la esencia que le corresponde en el complejo mundo de la información contemporánea, lo que nos conduce a un imprescindible, aunque breve, recuento histórico.

Los procesos de análisis y síntesis, corno base del tratamiento de la información primaria para obtener nuevos conocimientos, han sido practicados siempre por el hombre, porque son procesos del pensar. Consecuentemente Weisman 
(1972) afirma que el concepto de análisis de información es tan antiguo como la cultura humana y Horing (Ibidem) nos dice que cada individuo actúa como un centro de análisis de información, cuando usa el conocimiento existente para resolver o formular un problema. Estos procesos, potenciados por procedimientos de investigación cada vez más precisos, han sido utilizados durante siglos por los científicos de las más diversas ramas del conocimiento para obtener nuevos conocimientos sobre sus objetos de estudio. Hay esos procesos han llevado a una nueva división social del trabajo en los colectivos científicos y se han desplazado al campo de la información, aplicándose en ellos los recursos más modernos de la electrónica.

Todos sabemos que desde la antigüedad existieron documentos creados para el uso propio de sus autores o dueños, como testimonios de propiedad o de transacciones comerciales o estatales, que no fueron concebidos para su difusión.

Existieron también documentos destinados a fijar los conocimientos acumulados por la humanidad, trasmitidos oralmente hasta la aparición de la escritura, y que sirvieron, además, para fijar los productos de la imaginación y la creación de los hombres. Por la índole de sus contenidos estos documentos eran susceptibles de difusión.

Con el tiempo surgieron y se transformaron, pues, documentos que cumplen funciones sociales distintas (difusión / no difusión), o, al decir de Carrión (1988) documentos que pertenecen al conocimiento social y documentos que, por lo general, no pertenecen a éste.

El grado de difusión ha dependido siempre de los portadores, de su reproducción y, sobre todo, de las posibilidades de acceder a ellos. Esas posibilidades se han ido ampliando históricamente, pasando en lo tecnológico, por las etapas de reproducción manual, impresa y audiovisual hasta llegar a la electrónica en nuestros días con las posibilidades, al parecer infinitas, que ofrece la telemática. En lo social esa ampliación, aunque cierta, hoy corre serios peligros debido al aumento del0 analfabetismo en el mundo, a pesar de la denominada globalización de la información.

Los primeros ejemplos de descripción de documentos se sitúan alrededor del año 2000 a.n.e. (Mijailov, Chiorniii y Guiliarevskii, 1973). Estas descripciones se utilizaron en ocasiones para inventariar las colecciones mantenidas por una institución, pero en otras muchas sirvieron para integrar compilaciones no referidas a colecciones específicas. Las descripciones de documentos han evolucionado en forma y contenido hasta llegar a las actuales bases de datos bibliográficas computerizadas y en muchos casos es posible localizar, junto a la descripción o identificación $\mathrm{M}$ documento al documento mismo. 
Entonces, los procesos de análisis y síntesis, la acumulación de documentos de archivo o bibliotecas, diferenciados por su función social, y la descripción de documentos definen actividades informativas que han evolucionado históricamente en estrecha interrelación, pero que presentan rasgos esenciales propios.

En las postrimerías del siglo XIX el nivel alcanzado por las ciencias condujo a una crisis organizativo conceptual en esa esfera que se reflejé en el trabajo bibliográfico y en los servicios bibliotecarios especializados aparecidos durante ese siglo. la descripción de documentos y su organización reclamaban nuevos sistemas más flexibles e informativos (expresivos), acordes con las circunstancias.

De tal suerte se produjeron nuevos desarrollos en el campo de la Bibliografía, principalmente en el de la Bibliografía de temas científicos y técnicos. Surgieron nuevos sistemas de clasificación y la descripción de forma y contenido de los documentos se fue haciendo cada vez más profunda y precisa mediante la aplicación de distintos procedimientos.

La circulación de documentos, organizada por archivos y bibliotecas (como instituciones destinadas a acumular y facilitar el acceso a todo tipo de portador de información) se fue ampliando mediante el empleo de técnicas cada vez más avanzadas, reforzando al mismo tiempo las posibilidades de conservación.

De esta manera, el trabajo altamente especializado al servicio de ramas específicas de la ciencia, como actividad necesaria para el tratamiento de la información, incluida la reproducción de los materiales correspondientes recibió el nombre de documentación y ésta adquirieron la connotación de nueva disciplina académica. Diversos autores, con Paul Otlet como precursor, le han reconocido el status de disciplina científica integradora.

Pero a mediados del siglo XX se produjo una división del trabajo en la esfera de la investigación científica que modificó, la función de la documentación

Al científico teórico y al experimental se sumó el analista de información, a quien el autor soviético A. I. Mijailov llama informador (Mijailov, Chiorniii y Guiliarevskii, 1973). El analista es un científico que tiene la tarea de localizar, analizar y sintetizar la información existente con vistas a la solución de los problemas de investigación que aborda su colectivo. En estas circunstancias, las funciones de análisis y síntesis se desplazan al conjunto de actividades de la información.

Desde el punto de vista Informativo ya no era suficiente la función de "documentar". Se trataba entonces de obtener, de la información existente, una información cualitativamente nueva, capaz de resolver los problemas de investigación abordados, o, al menos, de precisar claramente sus límites y enunciados. 
La documentación a estas alturas reclamaba, no sólo conocimientos propios de los procesos bibliográficos, archivísticos y bibliotecarios, y de técnicas y tecnologías avanzadas, sino que exigía también el dominio del tema a tratar, el conocimiento de las regularidades de la información y la comunicación científica y de las técnicas de investigación que facilitan el análisis y la síntesis. En ese contexto, el término de documentación corno actividad fue sustituido en muchos lugares por el de actividad científico, informativa y, como disciplina, por el de ciencia o estudios de la información

Debe llamarse la atención sobre el hecho de que en la evolución antes descrita, la esencia de la descripción, acumulación, preservación y uso, propios de los procesos bibliográficos, archivísticos y bibliotecarios, se han mantenido vigentes en la documentación y en las aplicaciones tecnológicas más recientes. Para acceder a un documento vía internet, por ejemplo, es necesaria la aplicación de la telemática, pero es imprescindible que existan los elementos descriptivos del documento, convenientemente estructurados para localizarlo y recuperarlo; hace falta, además, que el propio documento esté incorporado al sistema, directamente o mediante su digitalización, para que pueda ser consultado en múltiples ocasiones por lectores diferentes. Esto imprime un nuevo enfoque al concepto clásico de 'circulación de documentos", al que algunos han dado en llamar "acceso o acceso remoto a la Información", pero no lo elimina, y lleva a la aparición del concepto de biblioteca virtual y, por qué no, de archivo virtual, puesto que los documentos de archivo y de bibliotecas se distinguen por la función social que desempeñan y según el grado de acceso que se permite a la información que contienen, pero ambos están sometidos a procesos de descripción, acumulación, preservación y uso. Nótese, entonces, que si el concepto de biblioteca virtual sugiere - aunque no parece posible hasta donde se avisora - la futura desaparición de la biblioteca tal cual la conocemos hoy, la esencia de la función bibliotecaria, sintetizada en la acumulación de documentos susceptibles de difusión y la preservación de esos documentos y/o sus contenidos, al tiempo que se facilita el acceso a ellos, se mantiene.

La complejidad actual del mundo de la información requiere la delimitación clara de sus componentes con el fin de lograr la profundización necesaria en el estudio de cada uno de ellos y para asumir ordenadamente las innovaciones que reclaman, al tiempo que se favorece la especialización sin olvidar la interrelación sistémica que existe entre todos los elementos de ese mundo.

Una forma conveniente para lograr esa delimitación es la de establecer cómo cada uno de los elementos que integran la complejidad informativa actual obtiene los productos fundamentales con que satisface las demandas de información y de establecer las diferencias cualitativas entre esos productos. 
Así es posible reconocer, en lo que se puede denominar estrategia racional de búsqueda informativa, al menos cuatro elementos con productos cualitativamente diferenciados, a los que se pueden adscribir disciplinas científicas interrelacionadas, pero con, objetos de estudio específicos y diferenciados. Esos elementos son: la actividad bibliográfica, cuyo producto fundamental es la compilación bibliográfica que da información sobre documentos, y es estudiada por la Bibliografología; la actividad archivistica, que tiene como producto fundamental la colección de archivo integrada por documentos que testifican, no concebidos generalmente para su difusión, y es estudiada por la Archivología; la actividad bibliotecaria, que produce la colección de biblioteca, integrada por documentos que trasmiten conocimientos y han sido, por lo general, objeto de difusión, estudiada por la Bibliotecología; la actividad científico informativa, que obtiene fun-

\begin{tabular}{|c|c|c|c|}
\hline Demandas & $\begin{array}{l}\text { Productos } \\
\text { fundamentales que } \\
\text { satisfacen las } \\
\text { demandas }\end{array}$ & $\begin{array}{l}\text { Etapas de la circulación } \\
\text { social de la información que } \\
\text { condicionan el producto }\end{array}$ & $\begin{array}{c}\text { Fenómeno } \\
\text { informativo } \\
\text { Disciplina que lo } \\
\text { estudia }\end{array}$ \\
\hline $\begin{array}{l}\text { de } \\
\text { información } \\
\text { sobre } \\
\text { documentos }\end{array}$ & $\begin{array}{l}\text { compilación } \\
\text { bibliográfica }\end{array}$ & & $\begin{array}{c}\text { actividad } \\
\text { bibliográfica } \\
\text { Bibliografología }\end{array}$ \\
\hline \multirow{2}{*}{$\begin{array}{c}\text { de } \\
\text { documentos } \\
\text { o de la } \\
\text { información } \\
\text { contenida en } \\
\text { ellos }\end{array}$} & $\begin{array}{l}\text { colección de archivo } \\
\text { (documentos que } \\
\text { testifican, no } \\
\text { concebidos en } \\
\text { principio para su } \\
\text { difusión) }\end{array}$ & creación de documentos & $\begin{array}{c}\text { actividad } \\
\text { archivística } \\
\text { Archívología }\end{array}$ \\
\hline & $\begin{array}{l}\text { colección de } \\
\text { biblioteca } \\
\text { (documentos que } \\
\text { transmiten } \\
\text { conocimientos, } \\
\text { susceptibles de } \\
\text { difusión) }\end{array}$ & difusión de documentos & $\begin{array}{c}\text { Actividad } \\
\text { bibliotecaria } \\
\text { Bibliotecología }\end{array}$ \\
\hline $\begin{array}{l}\text { de datos e } \\
\text { ideas } \\
\text { implícitos en } \\
\text { la } \\
\text { información } \\
\text { existente }\end{array}$ & información lógica & creación de información & $\begin{array}{l}\text { actividad científico } \\
\text { informativa } \\
\text { Ciencia de la } \\
\text { Información }\end{array}$ \\
\hline
\end{tabular}

Tabla 1. Estrategia racional de búsqueda informativa

Scire. $5: 1$ (en.-jun. 1999) 65-75. 
damentalmente, información lógica mediante los procesos de análisis y síntesis, estudiada por la Ciencia de la Información (Tabla I).

La incidencia de las nuevas tecnologías revoluciona hoy día la industria de la información y lleva a la proliferación de nuevos productos y servicios informativos. Entre estas actividades - la industrial y la de servicios - se produce una imbricación tal que, por momentos, parece que desaparecen los límites que les corresponden.

Lo sucedido en el mundo informativo durante el presente siglo condujo la aparición de la Ciencia de la Información, que para algunos es una ciencia abarcadora que engloba en la actualidad los contenidos que tradicionalmente han correspondido a la Bibliografía, la Archivología y la Bibliotecología, pero que abarca, además, el estudio de los procesos que corren a cargo de comunicadores, lingüistas, informáticos, etc., por lo que otros prefieren denominar a este campo del conocimiento corno ciencias o estudios de la información, en plural.

Lo cierto es que ser profesional de la información es una cualidad que corresponde a múltiples especialistas. Digamos, por ejemplo, que la Epistemología estudia la relación información-conocimiento; la Lingüística estudia la forma de expresar la información en lenguajes naturales y artificiales; la Cibernética estudia cómo modelar físicamente los procesos informativos; la Matemática cómo medir información; la Lógica cómo simbolizaría; la Sociología investiga las causas sociales que condicionan el desarrollo del fenómeno informativo, el impacto de ese fenómeno en la sociedad; la Pedagogía estudia cómo dosificar la información-conocimiento para su mejor apropiación; la Psicología estudia las motivaciones de la creación y uso de la información, los hábitos de ese uso. Así sucesivamente, pudiéramos citar otros casos. Sin embargo, estas disciplinas no clasifican como ciencias de la información aunque la incluyan como parte de su objeto de estudio.

Resulta claro, por tanto, que la información está asociada a diversos sectores de la actividad humana, dada su naturaleza multi e interdisciplinaria y multi e intersectorial.

En este contexto, entre los muchos enfoques de la Bibliotecología de hoy corno disciplina, existen tres que me gustaría comentar.

Uno de ellos (Rendon, 1997) la compara con la física de fines del XIX y principios del XX sobre la base de que los físicos de aquel entonces se encontraron con fenómenos que no respondían a la concepción tradicional de la materia, que estaban en contradicción con esa concepción, por lo que la materia se les escapaba de las manos y se perdía en un modelo matemático, lo que les llevó a expresar que la materia desaparecía y lo único que quedaba eran las ecuaciones. Según esta concepción en la Bibliotecología actual sucede algo similar, puesto que el

Scire. $5: 1$ (en.-jun. 1999) 65-75. 
objeto tradicional de la disciplina - considerando como tal al libro y a la biblioteca., al estilo de Buonocore- se escabulle, se transforma y se desplaza a otros fenómenos como documentos, bases de datos, redes, centros de documentación, bibliotecas electrónicas, ciberespacio, biblioteca virtual, y otros. Bajo este enfoque los profesionales de la Bibliotecología estamos en desventaja con los físicos de fines del siglo pasado porque a los bibliotecólogos no nos quedan ni siquiera ecuaciones matemáticas con qué trabajar

Sin embargo, los avances de la Bibliotecología en diferentes latitudes muestran que si aceptáramos que los físicos de fines del XIX y principios del XX se quedaron sólo con ecuaciones que modelaban estados concretos de la materia y no la generalidad de sus manifestaciones, los bibliotecólogos contemporáneos tenemos a nuestra disposición — si deseamos utilizarlo - el enunciado de una ley general del desarrollo del fenómeno bibliotecario que permite interpretar el pasado, analizar lo que sucede en el presente y proyectarse al futuro - lo que es imprescindible para enfrentar objetivamente las innovaciones que se nos enciman-, puesto que esa ley de carácter social, — que no ha sido representada matemáticamente, aunque quizá pueda serlo—, modela el fenómeno bibliotecario universalmente. ¿Qué es en realidad el fenómeno bibliotecario? ¿Cómo saber si existen similitudes entre la biblioteca de Asurbanipal y la biblioteca virtual a la que accedemos vía internet? ¿Es posible sustentar la permanencia del fenómeno bibliotecario en el próximo milenio? ¿Cuál será la misión del bibliotecario entonces? Precisamente, mediante la aplicación de la ley antes mencionada y de; aparato conceptual que la acompaña, es posible proponer respuestas a preguntas como estas. Respuestas que no son fáciles de obtener debido a la complejidad de los factores que intervienen en el desarrollo sujeto a ley del fenómeno bibliotecario y que reclama la necesidad de conocerlo esencial e históricamente y de estudiar profundamente la situación socioeconómica en la que se desenvuelve a cada época para poder evidenciar su interrelación con esos factores y encontrar las vías adecuadas para guiarlo en todo momento y, especialmente, en las actuales condiciones de innovación y cambios.

Por otra parte, en la Enciclopedia internacional de Bibliotecología y Ciencja de la información, publicada en Londres en 1997 (Enciclopedia..., 1997), al referirse a la ciencia de la información, se dice que nuestra esfera de conocimiento carece aún de una estructura teórica unificada (lo que es cierto), tal como se encuentra, por ejemplo, en el campo de las ciencias naturales. Según esa definición, estamos esperando todavía por un Linneo o un Mendeleev que resuelva esta situación.

Pienso que no es necesario esperar por un Mendeleev o un Linneo en Bibliotecología, porque ya llegaron hace años. Quizá no en una o dos personas, pero sí en un colegio invisible de bibliotecólogos cuyos aportes han permitido

Scire. $5: 1$ (en.-jun. 1999) 65-75. 


\section{2}

obtener conclusiones sobre la periodización de ; fenómeno bibliotecario y sobre la clasificación de sus elementos, pero, sobre todo, porque han establecido la forma de abordar esa periodización y esa clasificación en función de la dinámica social. Ocurre, sin embargo, que esos avances no se han generalizado y su aceptación es lenta, porque la inmensa riqueza del mundo fenoménico dificulta llegar a las esencias en nuestro caso. Lo cierto es, corno razonan Budd (1995) y Harris (1986) al analizar el enfoque hermenéutico fenomenológico de los problemas bibliotecarios e informativos, que las formas del pensar no se pueden trasladar mecánicamente de una esfera de la realidad a otra para la que no fueron concebidas. La clasificación de las especies de Linneo y la periodización de los elementos químicos de Mendeleev, responden al estudio de dos esferas distintas: la biológica y la química, y la aproximación al estudio de los fenómenos que a ellas corresponden es cualitativamente distinta. Con el método de Mendeleev no se pueden clasificar las especies, ni pronosticar su evolución, y con el de Linneo no es posible periodizar los elementos químicos ni prever el descubrimiento de otros. Esa imposibilidad es mucho mayor cuando se trata de abordar el análisis fenómenos sociales como el bibliotecario. De todas las esferas de la realidad, la social es la más dinámica debido a que en ella interviene el hombre con su voluntad transformadora. Consecuentemente, la clasificación de sus elementos se reajusta incesantemente y varían, incluso, sus denominaciones y hasta los criterios sobre su periodización. Por este proceso ha transitado el fenómeno bibliotecario y constituye una de las razones objetivas que subyacen, en parte, a las diferentes aproximaciones teóricas que enfrentamos en la profesión.

El sueco Rómulo Enmark (1998), en trabajo presentado a la Conferencia de IFLA en 1998, reconoce que la Bibliotecología es ya una disciplina por derecho propio, porque existen suficientes investigadores e informes de investigación en la rama, pero, fundamentalmente, porque existe un acuerdo tácito que reconoce su objeto de estudio a partir de ciertas estructuras de base institucional y de los procesos típicos de esas instituciones.

Sin embargo, los paradigmas institucionales, que identifican el contenido de la Bibliotecología con lo que sucede en las bibliotecas, han sido superados ampliamente al reconocerse, junto a los elementos esenciales del fenómeno bibliotecario, otros surgidos en distintos períodos históricos, que no se producen necesariamente en las bibliotecas, como la legislación bibliotecaria, las publicaciones bibliotecológicas, la formación profesional, el movimiento asociativo, la investigación bibliotecológica, la tecnología bibliotecaria y la teoría bibliotecológica, pero que forman parte indisoluble del fenómeno y, por tanto, del objeto de estudio de la Bibliotecología. Esto puede apreciarse en el análisis de las fases por las que ha discurrido el fenómeno bibliotecario.

Scire. $5: 1$ (en.-jun. 1999) 65-75. 
Actualmente la Bibliotecología, al igual que otras disciplinas relacionadas con la información, presenta, corno ya se dijo, diferencias teóricas debido a las distintas posiciones que se asumen ante la delimitación de su objeto de estudio y ante las formas de abordar ese objeto. Como ejemplo de esas tendencias se pueden citar:

1. Tendencias según el objeto de estudio que se le reconoce

1. La biblioteca (historia y funcionamiento)

2. La biblioteca (historia y funcionamiento) y el libro (producción y difusión)

3. Las leyes de circulación social del libro

4. El fenómeno bibliotecario (comprende la actividad bibliotecaria a partir de su esencia: lector-demanda, circulación, bibliotecario-colección; su historia y funcionamiento; interrelación con la sociedad la legislación bibliotecaria, las publicaciones bibliotecológicas, la formación del bibliotecario, sus asociaciones profesionales, la investigación bibliotecológica, la tecnología bibliotecaria, la teoría bibliotecológica)

5. La información (concepto con múltiples interpretaciones)

2. Tendencias según los contenidos que se le reconocen:

1. Paradigma docente (iguala contenido de la disciplina con los de planes de estudio de formación profesional)

2. Paradigma institucional (Iguala contenido de la disciplina con lo que sucede en la biblioteca)

3. Paradigma científico (con fundamento en la identificación de elementos esenciales e históricos, leyes y principios del fenómeno bibliotecario).

3. Tendencias según la esfera del conocimiento en que se inserta:

1. Ciencias técnicas (responde a paradigma tecnológico; deja en segundo plano, cuando más, la función social de la actividad).

2. Ciencias sociales y humanísticas (responde a paradigma del proceso de comunicación).

3. En la frontera de ambas.

4. Tendencias según escuelas filosóficas que la fundamentan:

1. La positivista en sus diferentes variantes

2. La marxista 
3. Otras con menor incidencia (pragmatismo, existencialismo, neotomismo, etc.).

5. Tendencias según sus límites:

1. Disciplina generalizadora (Ej. Bibliotecología abarca: Bibliología, Bibliografía, como en Buonocore)

2. Disciplina específica

3. Parte de un sistema de conocimientos del ámbito informativo

6. Tendencias según carácter que se le reconoce:

1. Disciplina técnica (no ofrece generalizaciones universales; explica procedimientos)

2. Disciplina científica (ofrece generalizaciones universales; enuncia leyes)

3. Disciplina de ambos caracteres: General si ofrece generalizaciones universales o enuncia leyes y aplicada si nos explica procedimientos.

Según nuestro criterio es preciso reconocer un conjunto de disciplinas estrechamente interrelacionadas, pero ni generalizadoras ni autosuficientes. Son disciplinas que se integran en un sistema que permite observar las relaciones que existen entre ellas y con otras ramas del saber.

Finalmente, debe reiterarse que vivimos en un mundo en plena fase de globalización y, como ya fue mencionado, de globalización de la información. Ese mundo trae problemas tremendos y desafíos inmensos. La necesidad de estar preparados para la gestión de innovación que se nos viene encima reclama conocimientos, cultura y, sobre todo, conciencia política y científica. El pensamiento bibilotecológico, como parte de la cultura y de la conciencia política y científica - aunque a veces no se le considere como tal—, no puede estar ajeno a esa realidad y se impone la reflexión teórica, de manera que podamos discernir entre lo que nos corresponde dentro de la tendencia normal a la internacionalización por la que marcha inexorablemente la humanidad, y lo que propone para la Bibliotecología y otras disciplinas informativas el carácter actual de la globalización.

La globalización atenta actualmente contra las culturas nacionales, a través de múltiples vías, incluida la información en sus formas más diversas. Atenta, consecuentemente, contra las identidades nacionales. Promueve una tendencia hacia una monocultura universal. Lo que se está promoviendo no es un mundo que combine la riqueza y la cultura de muchos países, sino un orden mundial que, por definición, destruye la cultura. Decididamente, no se puede concebir un mundo semejante, so pena de empobrecer su riqueza espiritual. Los profesionales de 
la información, incluidos los bibliotecarios, son comunicadores por definición y han de estar alertas ante esta situación en su gestión de innovación.

Esclarecer, mediante el análisis riguroso de las relaciones que mantienen con el medio el fenómeno bibliotecario, cómo las condiciones sociales inciden en él, cómo se inserta a su vez en la vida social, cómo está contribuyendo a su desarrollo, qué cambios se prevén en las condiciones sociales imperantes y cómo deben plantearse las perspectivas del fenómeno a la luz de esos cambios, constituyen tareas fundamentales del bibliotecólogo. ¿Cómo resolver los problemas que plantea el libre acceso a la información, de por sí contradictorio debido al crecimiento alarmante del analfabetismo en el mundo, frente a leyes cada vez más estrictas sobre el derecho de autor, apoyado por el concepto en boga de capital intelectual? ¿Cómo enfrentar la asimilación de tecnologías, cada vez más novedosas y bienvenidas, pero que imponen un ritmo de innovación altamente costoso, si se quiere estar al día? ¿Cómo abordar la preservación de la información ante las dudas que se esgrimen sobre la durabilidad de los nuevos portadores? ¿Cómo considerar el enfrentamiento entre la misión social del bibliotecario y el carácter empresarial que va tornando la actividad en virtud de las leyes ciegas de mercado que se nos imponen cada vez más? A mi juicio estas son algunas de las interrogantes que se deben resolver para una innovación y gestión de cambio en la actualidad, que garanticen un futuro más prometedor en los sistemas bibliotecarios

\section{Referencias}

Budd, M. (1995). An epistemological foundation for Library and Information Science. // Library Quaterly 65 : 3 (1995) 295-318.

Carrión, Manuel (1988). Manual de bibliotecas. Madrid : Pirámide, 1988.

Enmark, Romulo (1998). The point that doesn't exist : on a subject-defining concept of infomration. // Papers of the 64th IFLA Conference 1998. Booklet 7, p. 19-50.

Harris, Michael H. (1986). The dialectic of Defeat: antimonies in research in Library and Information Science. // Library trends. 34 (Spring 1986) 515-531

International Enciclopedia of Information and Library Science. London, 1997.

Mijailov, A. I ; Chiorniii, A. I. ; Guiliarevskii, R. S. (1973). Fundamentos de la Informática. La Habana : Academia de Ciencias de Cuba, 1973.

Rendon, Miguel Ángel (1997). Hacia un nuevo paradigma en Bibliotecología. // Transinformaçao. $8: 3$ (sept.-dic. 1996) 17-31.

Weisman, Herman W. (1972). Information systems, services and centers. New York : Wiley-Baker-Hayes, 1972. 